\title{
Photoinduced Signal Amplification Through Controlled Externally Sensitized Fragmentation in Masked Sensitizers
}

\author{
Rudresha Kottani, Janaki R. R. Majjigapu, Alexei Kurchan, Kavitha Majjigapu, Tiffany P. \\ Gustafson, and Andrei G. Kutateladze \\ Department of Chemistry and Biochemistry, University of Denver, Denver, CO 80208-2436.
}

Detection of molecular recognition events has always been an area of primary focus in bioanalytical sciences, with methods based on fluorescence being of preference due to their sensitivity. However, high throughput fluorescence binding assays require the tested ligands to be either spatially addressable or segregated on support beads for mechanical sorting. Solution phase libraries, either unsupported or immobilized on sub-micron carriers, present a challenge, as there were no direct methods to assay them. Our recent methodology for direct screening of solution phase libraries, encoded with photolabile tags, resolves this limitation. ${ }^{1}$ It uses dithiane-based molecular systems, capable of photoinduced fragmentation, but only in the presence of external sensitizers, i.e. the fragmentation in such systems is made contingent on a molecular recognition event, which brings the sensitizer in the proximity of the photolabile unit (Scheme 1).

The next logical question is whether a molecular recognition event can trigger not just one fragmentation, but rather set off a progression of photochemical events, releasing multiple copies of the encoding dithiane tags, and thus enhancing the sensitivity of detection. In this Communication we prove that such photoamplification can be achieved.

Non-PCR amplification of molecular recognition has been implemented via enzymatic catalysis, ${ }^{2 \mathrm{a}}$ polymerization, ${ }^{2 \mathrm{~b}}$ or massive liquid crystal reorientation ${ }^{2 \mathrm{c}}-$ all in a spatially addressable fashion. Amplification of electrophoretic tags via sensitized generation of singlet oxygen, with the effect localized due to limited diffusion of ${ }^{1} \mathrm{O}_{2}$, has also been proposed. ${ }^{3}$ Our amplification strategy is fundamentally different. Addition of lithiated dithianes to diaryl ketones - potential sensitizers - disrupts conjugation between the two aromatic moieties, effectively masking the sensitizer. The photosensitized fragmentation in such adducts releases more diaryl ketone, capable of sensitization. In the experiment shown in Figure 1, $3 \mathrm{~mol} \%$ of 4,4'-dimethoxybenzophenone was added to its dithiane adduct in acetonitrile and irradiated, while UV absorption of the solution was monitored at $360 \mathrm{~nm}$. A typical autocatalytic curve was obtained, indicating that photorelease of the masked ketone accelerated this bimolecular fragmentation. While this process resembles a chain reaction, with the released sensitizer carrying the chain, it is controlled better than radical polymerizations, as the photoamplification chain can be stopped and/or re-initiated at any time by removing or applying the UV source. The amplification uses a source of UV photons to amplify the amount of sensitizer, which often needs to be replenished in photoinduced ET reactions to compensate for irreversible reduction. Another outcome of the amplification is the mass release of dithianes, triggered with a very small amount of the initiator.

akutatel@du.edu.

Supporting Information Available

Experimental procedures and spectra. This material is available free of charge via the Internet at http://pubs.acs.org. 
In conjunction with spatial segregation on surfaces, this approach can be utilized for bioanalytical purposes; Scheme 2 illustrates this principal concept. The tested ligand is sparsely tethered to a surface, which displays the dithiane-masked sensitizer as a bulk material. Incubation with a receptor, modified with a tethered sensitizer, brings the sensitizer into the vicinity of adducts (A, red arrow). As irradiation commences, the initiator unmasks proximal sensitizers on the surface which, in turn, can sensitize the unmasking of their neighbors (B, red arrows) yielding more sensitizer. Ideally, the process is continued in expanding concentric ripples, until all of the sensitizer is unmasked. As a result, an amplified release of dithiane tags into solution takes place, allowing for detection with enhanced sensitivity by GCMS. When the ligands are spatially segregated from each other on discrete polymer beads or dendrimers, as in the one-bead-one-compound libraries, they can be encoded with 2-alkyl- substituted dithiane adducts for subsequent mass-discriminating analysis of the released tags.

Figure 2 shows how suitable the dithianes are for GCMS detection: the first derivative singleion monitoring (SIM) trace of six alkyl dithianes encoding decimal 207, binary 0011001111 , is obtained with 0.5 pmoles of each dithiane per injection $(\mathrm{S} / \mathrm{N}<10)$.

N-Hydroxysuccinimide esters of the masked 3-carboxybenzophenone, both for direct coupling (i.e. 3) and tethered via GABA (4) or 11-aminoundecanoic acid (5), were synthesized as shown in Scheme 3. Masked xanthones 6-7 were synthesized similarly.

To emulate a small amount of the initiator, brought in by a molecular recognition event, we planted $5 \%$ of free sensitizer on polystyrene high loading beads $\left(\mathrm{PS}-\mathrm{NH}_{2} 1.86 \mathrm{mmol} / \mathrm{g}\right.$, $200 \mu \mathrm{m})$. The rest of the surface material was the masked sensitizer. Actual loading was determined by sulfur elemental analysis to be $1.17 \mathrm{mmol} / \mathrm{g}$. The beads were suspended in $\mathrm{MeCN}$, purged with argon, irradiated with a $320 \mathrm{~nm}$ long pass filter, and dithiane release was monitored by GCMS. Figure 3 shows a typical release profile: dithiane concentration increases, reaching a maximum, followed by a slow decrease due to secondary photodegradation.

Control experiments were performed to rule out inter-bead sensitization and self-cleavage. For this, additional two types of beads were prepared, containing $(A) 100 \%$ immobilized adducts; and (B) 100\% immobilized sensitizer. Irradiation of a 50-50 mixture of beads $A$ and $B \operatorname{did}$ not release dithianes, suggesting no inter-bead sensitization. Secondly, the masked sensitizer was tested for direct fragmentation under the same irradiation conditions, because such nonsensitized cleavage undermines amplification, producing false positives, not triggered by molecular recognition. Ultimately, at a shorter wavelength, any adduct is capable of selfcleavage. Amplification is feasible if, at a given wavelength, the sensitized fragmentation is overwhelmingly more efficient than self-cleavage, i.e. $\epsilon_{k e t} \emptyset_{\text {sens }} \gg \epsilon_{a d d} \emptyset_{\text {self }}$, where $\epsilon_{k e t}$ and $\epsilon_{a d d}$ are the extinction coefficients of the free sensitizer and its adduct, $\epsilon_{s e n s}, \epsilon_{s e l f}$ - the quantum yields of the sensitized process and self-cleavage. Addition of dithianes to aromatic ketones disrupts conjugation, causing large blue shifts. Yet, some adducts retain significant absorption - in thioxanthones it is due to the surviving diphenylsulfide moiety - and quantum yields of self-cleavage remain rather high. ${ }^{4}$ The adducts of benzophenone-3-and xanthone-2-

carboxamides (beads A) showed no detectable self-cleavage when irradiated for 5 hrs above $320 \mathrm{~nm}$ at $9.5 \times 10^{19}$ photons $/ \mathrm{hr}$ (the sensitized reaction in solution is fully completed within 1.5 hours at this flux).

Unlike amplification in solution (Figure 1), dithiane release from the beads lacks the S-shaped autocatalytic curve. This is due to (i) unwanted reduction or cross-linking of the sensitizer in the polymeric matrix and (ii) the fact that the tethered freshly unmasked sensitizer can only unmask a few of its immediate neighbors, as the amplification front moves on. In solution, due to free diffusion, any sensitizer can carry the chain as long as it manages to escape the irreversible reduction. Thus xanthones are of preference, as the reduction potential of the triplet 
xanthone is higher than benzophenone's. ${ }^{5}$ Yet, xanthones are less prone to hydrogen abstraction and survive longer in reducing matrices. 6

Same amplification experiments with small amounts of planted sensitizer were carried out using PAMAM- $\mathrm{NH}_{2}$ G5 dendrimers. In several experiments, according to sulfur elemental analysis, 86 to 99 of 128 primary amino groups on dendrimer's surface were coupled with the methyldithiane adduct of xanthone-3-carboxylate. After this, 5-6 molecules of free sensitizer were tethered to the dendrimer surface and irradiated. In all cases amplified amounts of released dithianes were detected in solution, i.e. exceeding the amounts of the planted sensitizer. The dendrimers were also solubilized in micellar solutions of SDS to prove the amplification concept in an aqueous environment. The size of a particle in $30 \mathrm{mM}$ SDS was estimated to be $8.8 \mathrm{~nm}$ by pulse field gradient NMR. The micelle-free dendrimer in DMSO- $\mathrm{d}_{6}$ has an estimated size of $6.4 \mathrm{~nm}$, which implies that with aqueous detergent one micelle contains one dendrimer molecule. Irradiation, followed by extraction with hexane and GCMS analysis again revealed massrelease of dithiane tags.

To conclude, using micro and nano-sized polymeric supports we proved the concept of photoamplification on surfaces, whereby a sensitizer autocatalyzes its own release, concomitant with the release of encoding tags. Such amplified release can be made contingent on a molecular recognition event, offering a promising methodology for high throughput bioanalytical applications.

\section{Supplementary Material}

Refer to Web version on PubMed Central for supplementary material.

Acknowledgment

This research is supported by NIH (GM067655).

\section{References}

1. Kottani R, Valiulin RA, Kutateladze AG. Proc. Natl. Acad. Sci. USA 2006;103:13917. [PubMed: 16956977]

2. (a) Zhu L, Anslyn EV. Angew. Chem. Int. Ed 2006;45:1190.For recent review seeSikes, H.; Bowman, C.; Hansen, R.; Kuchta, R. PCT Int. Appl. 2006. WO 2006031248 (c) Clare BH, Abbott NL. Langmuir 2005;21:6451-6461. [PubMed: 15982053]

3. Singh, S.; Chan-Hui, P-Y. 2005. US6,949,347

4. Majjigapu JRR, Kurchan AN, Kottani R, Gustafson TP, Kutateladze AG. J. Am. Chem. Soc 2005;127:12458. [PubMed: 16144371]Non-sensitized self-cleavage in the adducts of fluorescent ketones is utilized in the release and report systems:

5. Lathioor EC, Leigh WJ. Photochem. Photobiol 2006;82:291. [PubMed: 16042506]

6. Kutateladze AG, Kottani R, Kurchan AN, Majjigapu JRR, Shirk SM. Phosph. Sulf. Silicon 2005;180:1379. 


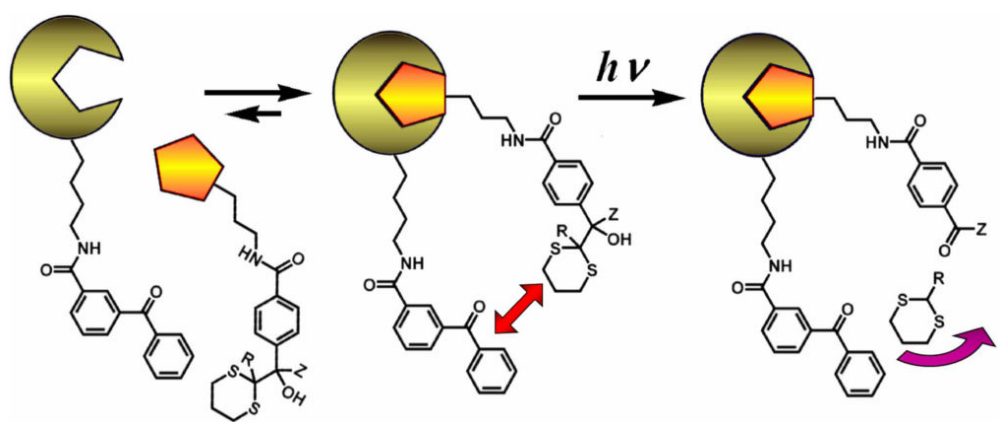

Scheme 1. 

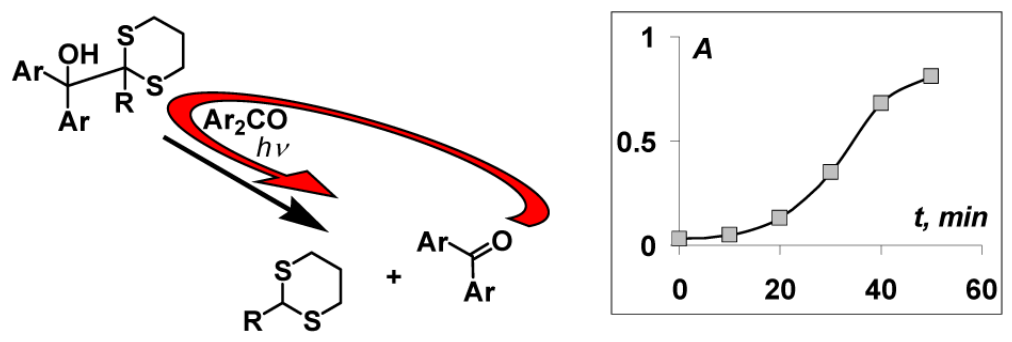

Figure 1.

Diarylketone sensitizing its own unmasking, as monitored by UV absorption @ $360 \mathrm{~nm}(\mathrm{Ar}=$ p-MeOPh-) 


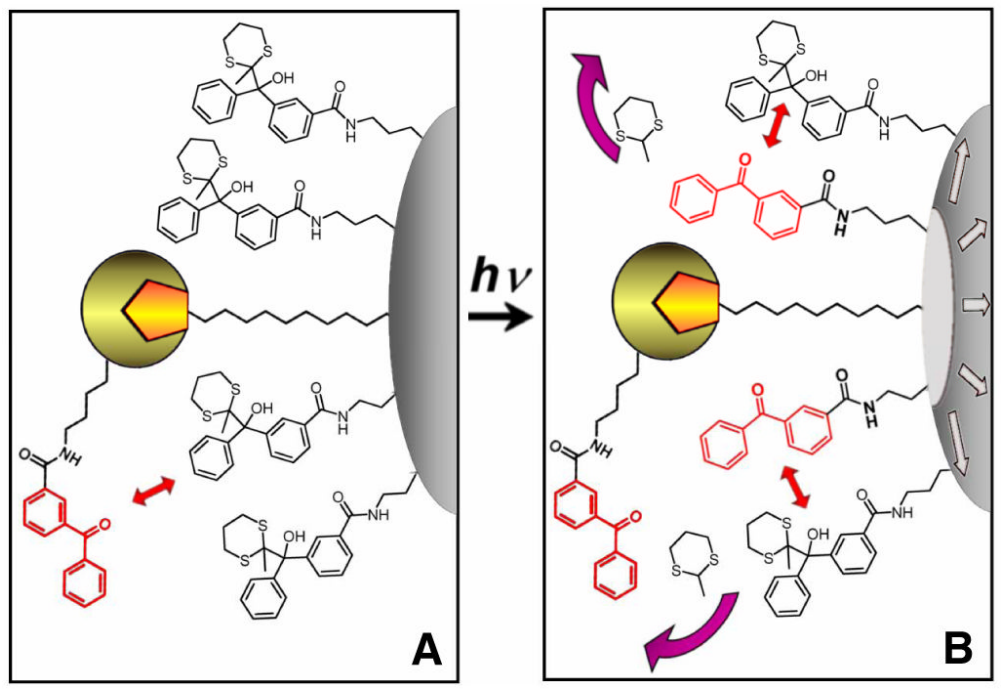

Scheme 2. 


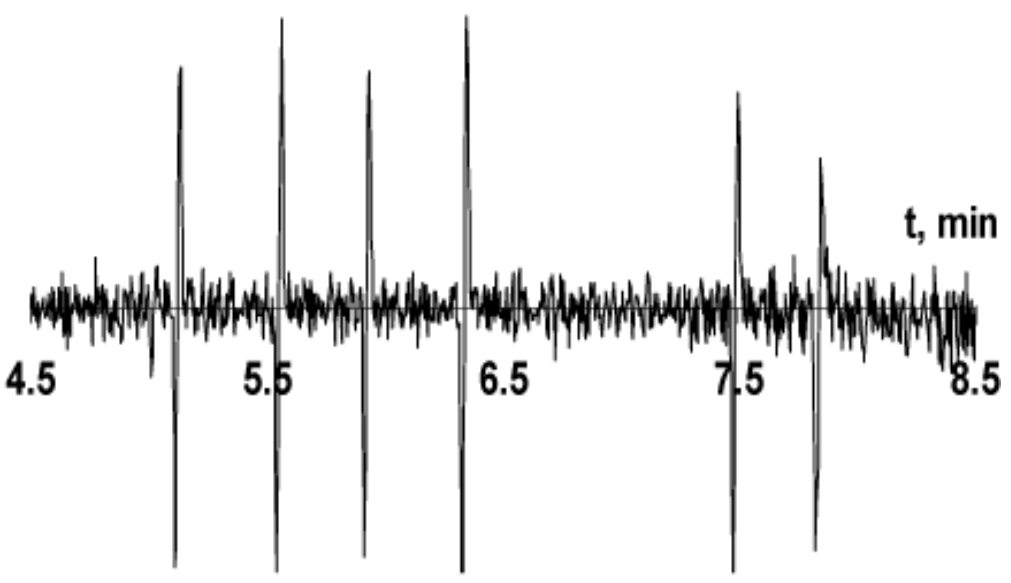

Figure 2.

SIM trace of 2-Me-, Et-, Pr-, Bu-, octyl- and nonyl-dithianes encoding decimal 207 


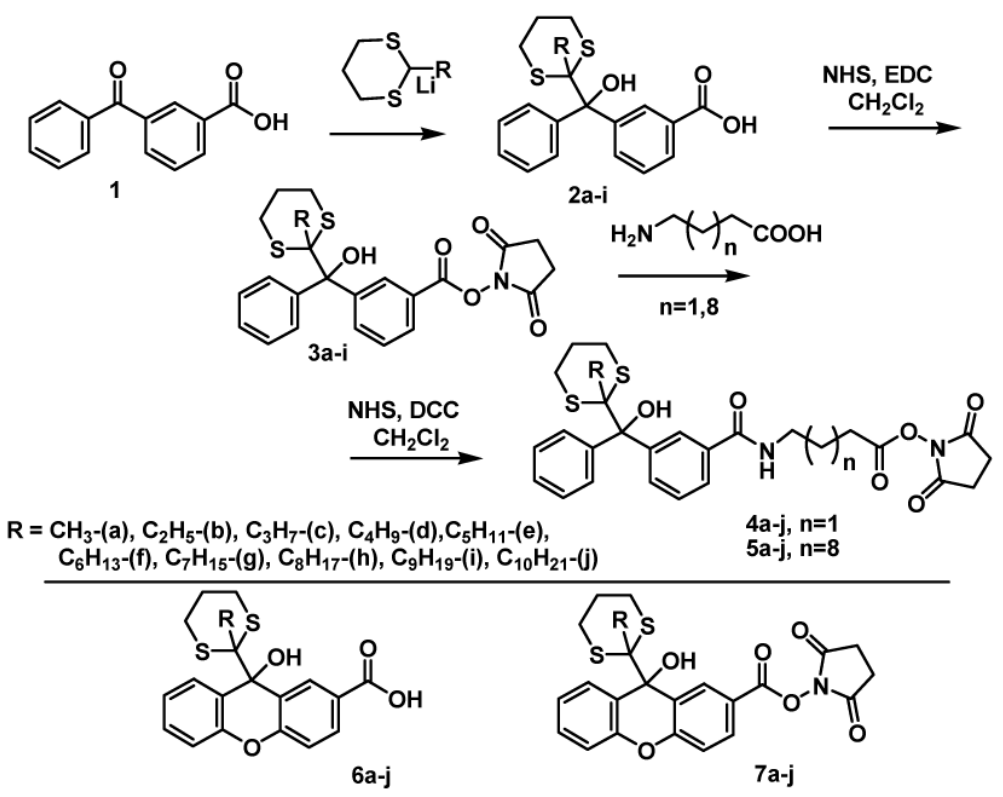

Scheme 3. 

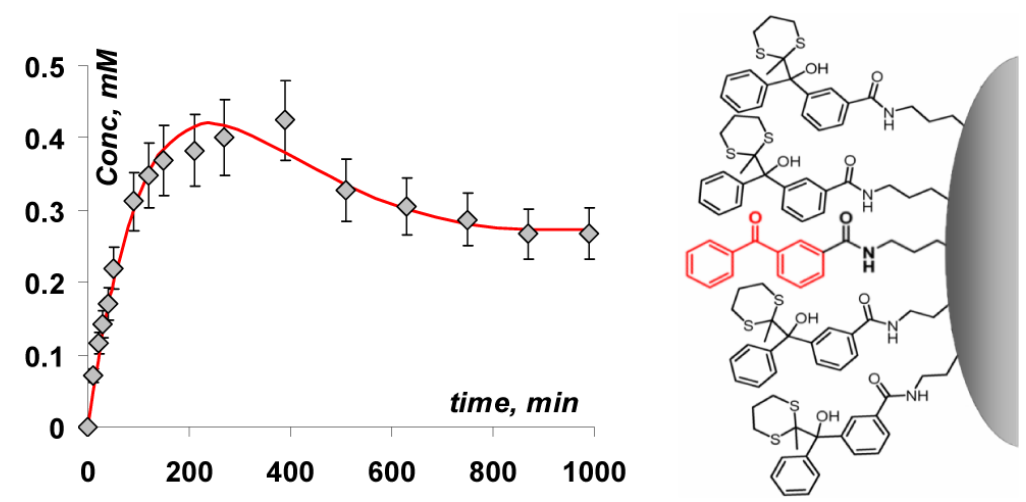

Figure 3.

Release of methyldithiane due to photoamplification of minute amounts of free sensitizer planted with the bulk of the masked benzophenone. 